\title{
The fittest, the common, and the dullest: Selection dynamics of exact autocatalytic replicators
}

\author{
Ben Shirt-Ediss ${ }^{1}$, Natalio Krasnogor ${ }^{1}$ and Harold Fellermann ${ }^{1}$ \\ ${ }^{1}$ Interdisciplinary Computing and Complex Biosystems Research Group, School of Computing, \\ Newcastle University, Newcastle-upon-Tyne NE4 5TG, UK \\ harold.fellermann@newcastle.ac.uk
}

\section{Extended Abstract}

In the origins of life field, one popular approach to frame and understand chemical evolution on the prebiotic earth is to focus on molecular replicators: in particular, to ask how complex and diverse molecular replicators could have arisen from a soup of simpler and less diverse species, without the aid of specific catalysts. Experimental work has demonstrated that template-directed, non-enzymatic replication of short oligonucleotides is indeed feasible (Joyce, 1984; Kiedrowski, 1986; Tjivikua et al., 1990). In these studies, a self-complementary nucleic acid template acts as an autocatalyst, creating identical copies of itself by hybridising short complementary strands, which subsequently ligate together with high probability and later de-hybridise from the original template (see reaction cycle in Fig. 1). This autocatalytic template replication scenario enables chemical sequence information to outlive the average life time of the individual molecules, thus giving rise to inheritable, and potentially selectable, information (Colgate and Ziock, 2011).

Recently, a minimal and mathematically tractable model of this chemical process was proposed (Tanaka et al., 2014; Fellermann et al., 2013) based on (i) binary strings able to catalyse their own formation by concatenating two matching substrings (Fig 1, reaction rule 2) and additionally (ii) the rare non-catalysed concatenation of any two strings, together with random degradation of a string into any two substrings, permitting an exploration of replicator sequence space (Fig 1, reaction rule 1). Analysis of this simple exact replicator model revealed several counter-intuitive findings, most notably the existence of a selection pressure for a few sequences that feature repeated short motifs, such as alternating monomers (...010101...) or arrangements in blocks of two (...00110011...). Fellermann et al. (2017) attribute this "survival of the dullest" effect to a cascade of symmetry breaks which is driven by the continued competition of emerging replicators. Moreover, Fellermann et al. (2017) show that this selection pressure can also be observed in several variants of the original model, including the use of bigger monomer alphabets, the possibility for point mutations, inert food species, as well as the scenario where templates

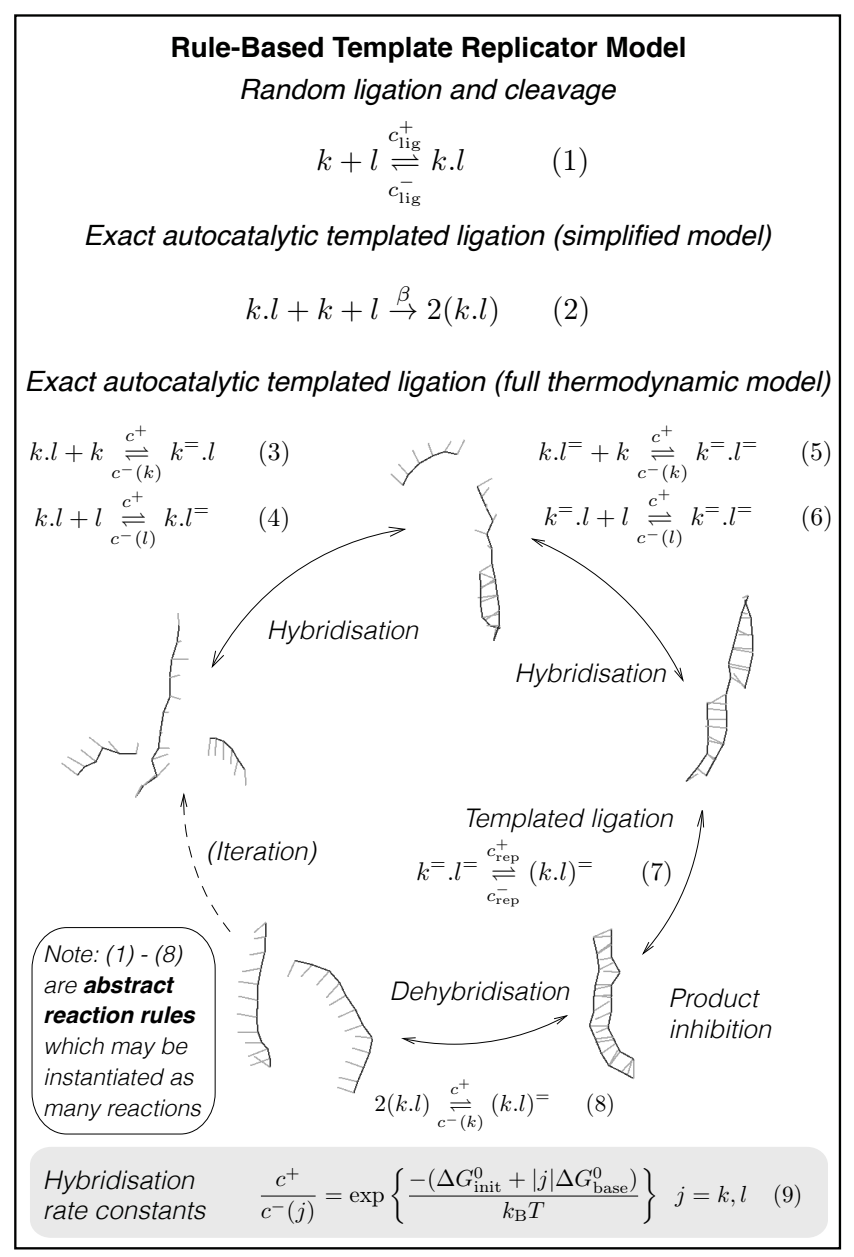

Figure 1: A simplified exact autocatalytic replicator model (reaction rules 1 and 2) is extended in this work into a more detailed model. Trimolecular templated ligation rule 2 is replaced with a series of reversible elementary abstracted reaction rules (3-8) whose rate constants are related by the free energy change of each respective reaction (equation 9). As the model is rule-based, $k$ and $l$ don't represent single species, but represent any polymer over a monomer alphabet $\left(\forall k, l \in\{0,1\}^{+}\right) .|j|$ denotes the length of polymer $j$. 
catalyze the ligation of complementary rather than identical sequences. All these variants exhibit survival of the dullest with slight differences in the selected sequences.

However, the original exact autocatalytic replicator model and all its studied variants utilize a single tri-molecular and irreversible replication reaction. What has not been answered at this point is the question whether "survival of the dullest" would continue to be observed in models of polymer templated replication that capture physico-chemical aspects of the involved chemical species with higher fidelity.

Inclusion of physico-chemical details could be important to the resulting replicator evolutionary dynamics. For example, it has been empirically observed that template directed replicators do not always feature the typical exponential growth curves known from autocatalysts, but can instead follow a slower growth curve, where the growth rate of the replicator is not directly proportional to its concentration, but rather to the square root of the concentration (Kiedrowski, 1993; Fellermann and Rasmussen, 2011). This "parabolic growth" effect is caused by product inhibition wherein a newly produced template strand binds with another template strand, making it inaccessible to the substrate and reducing the effective template concentration. Szathmáry and Gladkih (1989) signalled that parabolic growth can have important consequences in replicator ecologies: they analytically derived that exponential growth paired with resource competition leads to Malthusian survival of the fittest (and only the fittest) whereas parabolic growth leads to a qualitatively different scenario, survival of the common, where all competing replicators will survive a differential selection pressure, with abundances given by their relative replication rates. It is an open question whether parabolic replicators are equally affected by a selection pressure toward simple "dull" repetitive sequences, or whether their evolutionary dynamics could indeed enable a unconstrained exploration of the potential sequence space.

Here we present a refinement of the original exact replicator model, where the irreversible tri-molecular template ligation reaction (Fig. 1, reaction rule 2) is replaced by a series of elementary bi-molecular and reversible reactions (Fig. 1, reaction rules 3-8). Thus, the assumption of exponential autocatalysis of replicators is replaced by a more realistic scenario including product inhibition, and thus provides the possibility for parabolic growth of replicators. Furthermore, the reaction rate constants are not assigned arbitrarily, but are instead set so that they are consistent with an elementary nucleic acid energy model (SantaLucia, 1998), rendering the the resulting overall model thermodynamically consistent.

We implement this model as a rule based stochastic process where applicable reactions for a given system state are derived from the defining reaction rules during each step of a stochastic simulation algorithm. This allows us to sample the stochastic process without the need to constrain, e.g., the number of species or the maximal polymer length.
We are currently investigating whether "survival of the dullest" is still observed in this replicator model, and whether and how the different growth regimes affect the selection dynamics. After characterizing the equilibrium state of the model for relevant parametrizations, we study the dynamics of the system when driven out of equilibrium, namely (i) by providing an energy inflow to generate a temperature cycle, and (ii) in continuous flow stirred reactor conditions where a material inflow of "food" molecules in the form of monomers or short oligomers exists.

\section{Acknowledgements}

This work is supported by EPSRC Grant EP/N031962/1.

\section{References}

Colgate, S. A. and Ziock, H. (2011). A Definition of Information, the Arrow of Information, and its Relationship to Life. Complexity, 16(5):54-62.

Fellermann, H. and Rasmussen, S. (2011). On the growth rate of non-enzymatic molecular replicators. Entropy, 13(10):18821903.

Fellermann, H., Rasmussen, S., and Tanaka, S. (2013). Selection, stability and cooperation in a binary polymer model. In Europ Conf. on Complex Systems, Vienna, Austria.

Fellermann, H., Tanaka, S., and Rasmussen, S. (2017). Sequence selection by dynamical symmetry breaking in an autocatalytic binary polymer model. Phys. Rev. E, 96(6):062407.

Joyce, G. F. (1984). Non-enzyme template-directed synthesis of RNA copolymers. Orig. Life Evol. Biosph., 14:613-620.

Kiedrowski, G. v. (1986). A Self-replicating hexadeoxynucleotide. Angew. Chem., 25(10):932-935.

Kiedrowski, G. v. (1993). Minimal Replicator Theory I: Parabolic Versus Exponential Growth. In Bioorganic Chemistry Frontiers, Bioorganic Chemistry Frontiers, pages 113-146. Springer, Berlin, Heidelberg.

SantaLucia, Jr., J. (1998). A unified view of polymer, dumbbell, and oligonucleotide DNA nearest-neighbor thermodynamics. Proc. Nat. Acad. Sci. USA, 95:1460-1465.

Szathmáry, E. and Gladkih, I. (1989). Sub-exponential growth and coexistence of non-enzymatically replicating templates. $J$. Theor. Biol., 138(1):55-58.

Tanaka, S., Fellermann, H., and Rasmussen, S. (2014). Structure and selection in an autocatalytic binary polymer model. Europhys. Lett., 107(2):28004.

Tjivikua, T., Ballester, P., and Rebek, J. (1990). Self-replicating system. J. Amer. Chem. Soc., 112(3):1249-1250. 